\title{
Case - Fungal urosepsis after ureteroscopy in a patient on new generation of anti-hyperglycemic medication
}

Hamidreza Abdi; Eric Locke; Ryan Fitzpatrick; Jeffery Stuart Oake

Department of Surgery, Division of Urology, University of Ottawa, Ottawa, ON, Canada

Cite as: Can Urol Assoc J 2018 November 20; Epub ahead of print. http://dx.doi.org/10.5489/cuaj.5635

Published online November 20, 2018

$* * *$

\section{Introduction}

Diabetes mellitus is a growing disease with extensive health risks. ${ }^{1}$ Gliflozin represents a new class of medications in the treatment armamentarium for diabetes. Medications in this class include dapagliflozin, canagliflozin, and empagliflozin. Their primary mechanism of action is blocking sodium-glucose transporter protein 2 (SGLT)-2, which inhibits the reabsorption of glucose in the kidney, and therefore lowers systemic blood sugar levels. ${ }^{2}$ SGLT-2 inhibitors are known to have higher urinary glucose levels as a byproduct of lowering blood glucose levels. Not surprisingly, patients on gliflozins have an increased incidence of urinary tract infections, likely due to the associated glucosuria from the medication. ${ }^{3}$

Endoscopic urological interventions, particularly ones involving stone treatment, have a welldocumented risk for causing urinary tract infections. Prophylactic antibiotics are typically given in accordance with CUA guidelines and local resistance patterns to help reduce the risk of postoperative infections. ${ }^{4}$ Elevated urinary glucose levels can potentially increase the likelihood of post-endourological procedure infection. Urologists should, therefore, be aware of the higher risk of postoperative infections in patients on these novel agents and tailor their prophylactic antibiotics appropriately. Here, we report a case of a patient with Type 2 diabetes mellitus who returned to hospital five days following an uneventful ureteroscopy with sepsis.

\section{Case report}

A 59-year old male presented to the emergency room with renal colic secondary to a distal $10 \mathrm{~mm}$ ureteric stone detected on CT scan. His past medical history was remarkable for recurrent nephrolithiasis, previously treated with extracorporeal shock wave lithotripsy, gastroesophageal reflux, dyslipidemia, and diabetes mellitus (type 2). His medications included pantoprazole 40 mg P.O daily, metformin 500mg P.O. BID, Jardiance (empagliflozin) 25mg P.O. daily, and insulin (Lantus) $44 \mathrm{U}$ QHS. His usual glucoscan readings were between 5-6 mmol/L. He did not have a pre-op urine culture; however, his urinalysis was negative for nitrites and leukocyte 
esterases. He was hemodynamically stable, but his pain could not be controlled with medications. He was subsequently admitted and underwent an uneventful right ureteroscopy, laser lithotripsy and insertion of right double J ureteric stent. The patient received two grams of intravenous Ancef (Cefazolin) for prophylaxis as per our institutions antibiogram recommendations. He was discharged home later that day on a short course of ciprofloxacin postoperatively with a plan to remove the stent in 2 weeks.

Five days following the procedure, he presented to the emergency room with a 2-day history of a high-grade fever, chills, and nausea/vomiting. He denied any frequency, urgency or flank pain. He was febrile, tachycardic and mildly hypotensive, so the sepsis protocol was initiated. He was treated with intravenous (IV) fluids, Piperacillin-Tazobactam 3.375 gram, IV, every 6 hours and Vancomycin 1 gram IV every 12 hours. These were continued despite no major clinical improvement changes until blood cultures and urine cultures came back positive with yeast. No bacterial growth was identified. Fluconazole 400mg IV daily was initiated and the Infectious Diseases (ID) service was consulted.

He underwent a local cystoscopy to remove the stent as the ID team was worried the foreign body may harbour the fungal infection and make it difficult to eradicate. His fluconazole was switched to caspofungin $50 \mathrm{mg}$ IV daily. He showed dramatic clinical improvement and the repeat cultures returned negative for yeast. He underwent a full fungal work up as a result of the positive blood cultures, including a trans-thoracic echocardiogram and ophthalmology consultation. Both assessments were negative for any fungal complications. The speciation finally returned revealing Candida Glabrata species (spp.). He was discharged from hospital 10 days after admission, and he completed 4 weeks caspofungin $50 \mathrm{mg}$ IV daily via peripherally inserted central catheter (PICC) line followed by oral fluconazole $800 \mathrm{mg}$ daily for 2 more weeks. His infection fully resolved without any further issues.

He has been seen several times in follow up since. Eight months later, he developed another non-obstructing stone in the lower pole of left kidney, treated with an uneventful ureteroscopy and laser lithotripsy. He had a negative pre-operative urine culture and was not placed on any specific anti-fungal prophylaxis; however, he was encouraged to be well hydrated before and after the procedure. His stent was removed two weeks after ureteroscopy, and he had no further issues with infections.

\section{Discussion}

According to Canadian statistics, in 2015 it was estimated that 3.4 million Canadians (9.3\% of population) have diabetes. This is projected to increase to $44 \%$ by $2025{ }^{1}$ Traditional medications are often associated with adverse effects, increasing insulin resistance and deterioration of pancreatic B-cell insulin production with the disease progression. ${ }^{2,5}$ A novel class of medications called Gliflozins have demonstrated impressive results in lowering blood glucose level with no direct effects on pancreatic B-cell function. ${ }^{3}$ 
SGLT-2 inhibitors target the proximal convoluted tubules in the kidney and inhibit glucose reabsorption. This increases urinary glucose excretion, resulting in anti-hyperglycaemic effects. ${ }^{2}$ Empagliflozin (Jardiance) is a selective SGLT-2 inhibitor reported reducing glycated hemoglobin levels in patients with Diabetes Mellitus Type II. It has also reported additional benefits of reducing body weight, blood pressure, and cardiovascular events. ${ }^{6-9}$

Increased urinary glucose level causes increased urinary frequency with electrolyte imbalance. Glucosuria associated with SGLT-2 inhibitors has also been shown to increase the risk of genital and urinary tract infections including mycotic infection in various studies. ${ }^{3,9-12}$

Overall, fungal urinary tract infections, particularly Candida spp. and Aspergillus spp. , have been becoming increasingly common, due to the increased overall use of antibiotics, as well as the increasing prevalence of immune-deficient states, including diabetes. ${ }^{14,15}$ In the study by Tong et al. analysis of clinical data of patients on dapagliflozin showed that female gender and a previous history of infections were independent risk factors for genital fungal infections. ${ }^{16}$ Higher urinary glucose level could provide the yeast with more available nutrients, which can increase urine acidity and facilitate their overgrowth natural surfaces. ${ }^{18}$ There is no current literature on the risks of post-operative urinary tract infection rates while patients are on SGLT-2 inhibitors.

Any urologic intervention has a potential risk of urinary tract infection due to the effect of instrumentation, changes in the host defensive mechanisms, the breakdown of natural barriers and introducing bacteria into the urinary tract. ${ }^{17}$ Ureteroscopy is one of the most common urologic procedures, and with a growing number of diabetic patients on new anti-hyperglycemic medications, urologists should be aware of the higher risk of post-operative infections, including fungal infections.

In our case, the patient had diabetes and urinary tract disease which are two risk factors for funguria, although the blood glucose level was within the normal range. The optimal strategy to prevent this situation is not clear. Current Canadian guidelines recommend antibiotic prophylaxis for ureteroscopy and other endoscopic procedures, but there is not current recommended prophylaxis with antifungals. ${ }^{4}$ However, we believe, given the higher concentration of urinary glucose in patients on SGLT-2 inhibitors, it may be reasonable to consider hydration perioperatively and anti-fungal prophylaxis. The risk-benefit ratio of this decision needs to be borne out in future studies. Our case highlights the importance of being aware of the side effects of all medications that our urologic surgical patients are taking, and what risk they can pose preoperatively, intra-operatively and post-operatively. This will allow us as urologists to maximize our patients' outcomes, and better identify and appropriately treat any complications that occur.

\section{Conclusion}

SGLT-2 inhibitors, a new generation of anti-hyperglycemic medications, may put our patients at higher risk of urinary tract infections and any urologist should consider this while planning for 
endoscopic procedures. Future well designed studies are needed to show the best preventative measures before urologic procedures in patients on this class of medications. 


\section{References}

1. Canadian Diabetes Association. (2015). Report on diabetes: Driving change. Toronto, ON: CDA.

2. Gallo L, Wright E, Vallon V. Probing SGLT2 as a therapeutic target for diabetes: Basic physiology and consequences. Diab Vasc Dis Res 2015;12:78-89.

3. Halimi S, Verges B. Adverse effects and safety of SGLT-2 inhibitors. Diabetes Metab J 2014;40:28-34.

4. Mrkobrada M, Ying I, Mokrycke S, et al. CUA Guidelines on antibiotic prophylaxis for urologic procedures. Can Urol Assoc J. 2015 Jan-Feb;9(1-2):13-22.

5. Campbell R. Fate of the beta-cell in the pathophysiology of type 2 diabetes. $J$ A Pharm Assoc 2009;49:10-5.

6. Grempler R, Thomas L, Eckhardt M, et al. Empagliflozin, a novel selective sodium glucose cotransporter-2 (SGLT-2) inhibitor: Characterisation and comparison with other SGLT-2 inhibitors. Diabetes Obes Metab 2012;14:83-90.

7. Zinman B, Wanner C, Lachin J, et al. Empagliflozin, cardiovascular outcomes, and mortality in type 2 diabetes. N Engl J Med 2015;373:2117-28.

8. Haring H, Merker L, Seewaldt-Becker E, et al. Empagliflozin as add-on to metformin plus sulfonylurea in patients with type 2 diabetes: A 24-week, randomized, double-blind, placebo-controlled trial. Diabetes Care 2013;36:3396-404.

9. Singh M, Kumar A. Risks associated with SGLT2 inhibitors: An overview. Current Drug Safety 2018;13:84-91.

10. Arakaki R. Sodium-glucose cotransporter-2 inhibitors and genital and urinary tract infections in type 2 diabetes. Postgraduate Med 2016;128:409-17.

11. Li D, Wang $\mathrm{T}$, Shen $\mathrm{S}$, et al. Urinary tract and genital infections in patients with type 2 diabetes treated with sodium-glucose co-transporter 2 inhibitors: A meta-analysis of randomized controlled trials. Diabetes Obes Metab 2017;19:348-55.

12. Levine J. Empagliflozin for type 2 diabetes mellitus: An overview of phase 3 clinical trials. Curr Diabetes Rev 2017;13:405-23.

13. Rizzi M, Trevisan R. Genitourinary infections in diabetic patients in the new era of diabetes therapy with sodium-glucose cotransporter-2 inhibitors. Nutr Metab and Cardiovasc Dis 2016;26:963-70.

14. Perlroth J, Choi B, Spellberg B. Nosocomial fungal infections: Epidemiology, diagnosis, and treatment. Medical Mycology 2007;45:321-46.

15. Rao P. Aspergillus infection in urinary tract post-ureteric stenting. Indian $J$ of Med Microbiol 2015;33:316-8.

16. Thong K, Yadagiri M, Barnes D, et al. ABCD Nationwide Dapagliflozin Audit contributors. Clinical risk factors predicting genital fungal infections with sodium-glucose cotransporter 2 inhibitor treatment: The ABCD nationwide dapagliflozin audit. Primary Care Diabetes 2018;12:45-50.

17. Grabe M, Botto H, Cek M, et al. Preoperative assessment of the patient and risk factors for infectious complications and tentative classification of surgical field contamination of urological procedures. World J Urol 2012;30:39-50. 
18. Maalouf NM, Cameron MA, Moe OW, Sakhaee K. Metabolic Basis for Low Urine pH in Type 2 Diabetes. Clinical Journal of the American Society of Nephrology: CJASN. 2010;5(7):1277-1281. 